\title{
The variable gamma-ray sky seen by the Fermi LAT Flare Advocates
}

\section{Stefano Ciprini*}

(on behalf of the Fermi LAT collaboration)

ASI Science Data Center, Frascati, Roma, Italy

INAF Observatory of Rome, Monte Porzio Catone, Roma, Italy

E-mail: stefano.ciprini@asdc.asi.it

\begin{abstract}
The Fermi Flare Advocate (also known as Gamma-ray Sky Watcher, FA-GSW) service provides for a daily quicklook analysis and review of the high-energy gamma-ray sky seen by the Fermi Gamma-ray Space Telescope. Fermi is demonstrating good capabilities in gamma-ray variability monitoring and the FA-GSWs duty is complementing this all-sky surveying offering alerts for potentially new gamma-ray sources, transients and relevant flares. A public weekly digest is published in the web-based Fermi Sky Blog. During the first 3 years of all-sky survey, more than 150 Astronomical Telegrams, several alerts to the TeV Cherenkov telescopes and several targets of opportunity to Swift and other observatories have been realized. This increased the rate of simultaneous multi-frequency observing campaigns and the level of international cooperation with the Fermi mission.
\end{abstract}

The Extreme and Variable High Energy Sky - extremesky2011,

September 19-23, 2011

Chia Laguna (Cagliari), Italy

${ }^{*}$ Coordinator of the Fermi LAT FA-GSW service and the Fermi team at ASDC. 


\section{Gamma-ray variability monitoring and scope of the FA-GSW service}

The Large Area Telescope (LAT), on board the Fermi Gamma-ray Space Telescope is a pairconversion $\gamma$-ray telescope, sensitive to photon energies from about $20 \mathrm{MeV}$ up to $>300 \mathrm{GeV}$. The LAT is composed of a tracker (two sections, front and back), a calorimeter and an anti-coincidence system to reject the charged-particle background (Atwood et al., 2009). Fermi LAT, working in continuous all-sky survey mode, is an optimal hunter for high-energy bright flares, transients and new $\gamma$-ray sources, and is an unprecedented monitor of the variable $\gamma$-ray sky, thanks to the large peak effective area, wide field of view $(\approx 2.4 \mathrm{sr})$, improved angular resolution and sensitivity.

Irregular and aperiodic variability is found in about $2 / 3$ of the brightest Fermi LAT blazars. High states are less than 1/4 of the light curve range (Abdo et al., 2010a) and Flat Spectrum Radio Quasars (FSRQs) and low energy peaked BL Lac objects (BL Lacs) show the largest relative variance (Fig. 1). Different light curves and flare patterns, autocorrelation time scales and power spectrum (PSD) indices $\left(1 / f^{\alpha}\right.$ trends, where $\left.f=1 / t\right)$ are found implying different variability modes for each source (more flicker, $\alpha \simeq 1$ or more Brownian, $\alpha \geq 2$, dominated, Fig.1, not always correlating with $\gamma$-ray luminosity and blazar subfamily). No evidence for persistent characteristic $\gamma$-ray timescale(s) is found. Flare profiles are mostly symmetric (in part because of superimposing flares and large bin smoothing). The fractional variability during outburst appears similar to its longer term mean in the few objects studied in detail. A few more details are reported in the caption of Fig.1.

The Fermi LAT all-sky variability monitoring is complemented by the Flare Advocate (a.k.a. Gamma-ray Sky Watcher, FA-GSW) duty, a scientific service belonging to the LAT Instrument Science Operations and devoted to quicklook inspection and daily review of the gamma-ray sky
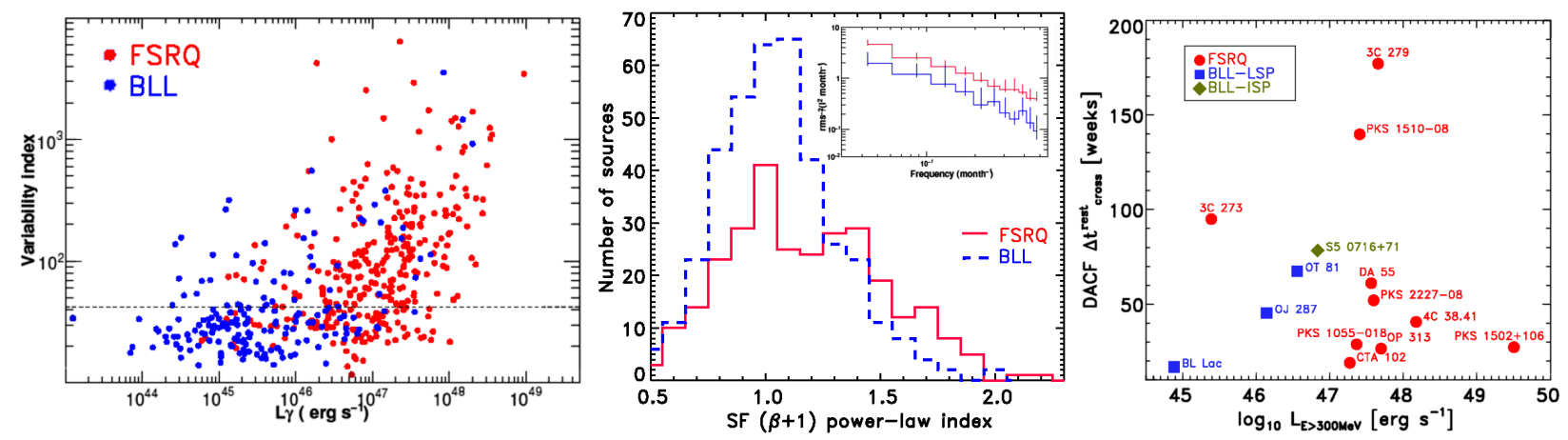

Figure 1: Results from $\gamma$-ray light curves extracted in fixed 1-month bins over the first 2 years of Fermi LAT all-sky survey for the 886 blazars/AGNs of the clean source sample of the second Fermi LAT Source Catalog, 2FGL. Left panel: variability index versus isotropic $\gamma$-ray luminosity (red/grey: FSRQs; blue/black: BL Lac Objects). Dashed line represents the $99 \%$ confidence level for a source to be variable. Central panel: distribution of the temporal PDS power-law indexes $(\alpha=\beta+1)$ for the FSRQs (red/continuous line) and BL Lac objects (blue/dashed line) of the sample evaluated in time domain using first order structure function (SF) analysis (blind power-law index $\beta$ estimation using a maximum lag of $2 / 3$ of the total light curve range). Cumulative power spectral density (PSD) for bright FSRQs (red/top line) and BL Lac objects sub-samples (blue/bottom line) showing similar slopes (inset). From Ackermann et al. (2011). Right panel: scatter plot of the weekly-binned variability auto-correlation (DACF) crossing times in the rest frame of the source (corrected for $\mathrm{z}$ and beaming) versus the total apparent isotropic $\gamma$-ray luminosity $(\mathrm{E}>300 \mathrm{MeV})$ in the co-moving frame for 15 bright LAT blazars that are also part of the MOJAVE program. 3C 454.3 is out of the plot range (with very long $\Delta t_{\text {rest }}^{\text {cross }}=254.3$ weeks, i.e. very Brownian behaviour, and $\log _{10}\left(L_{E}\right)=48.1$. 


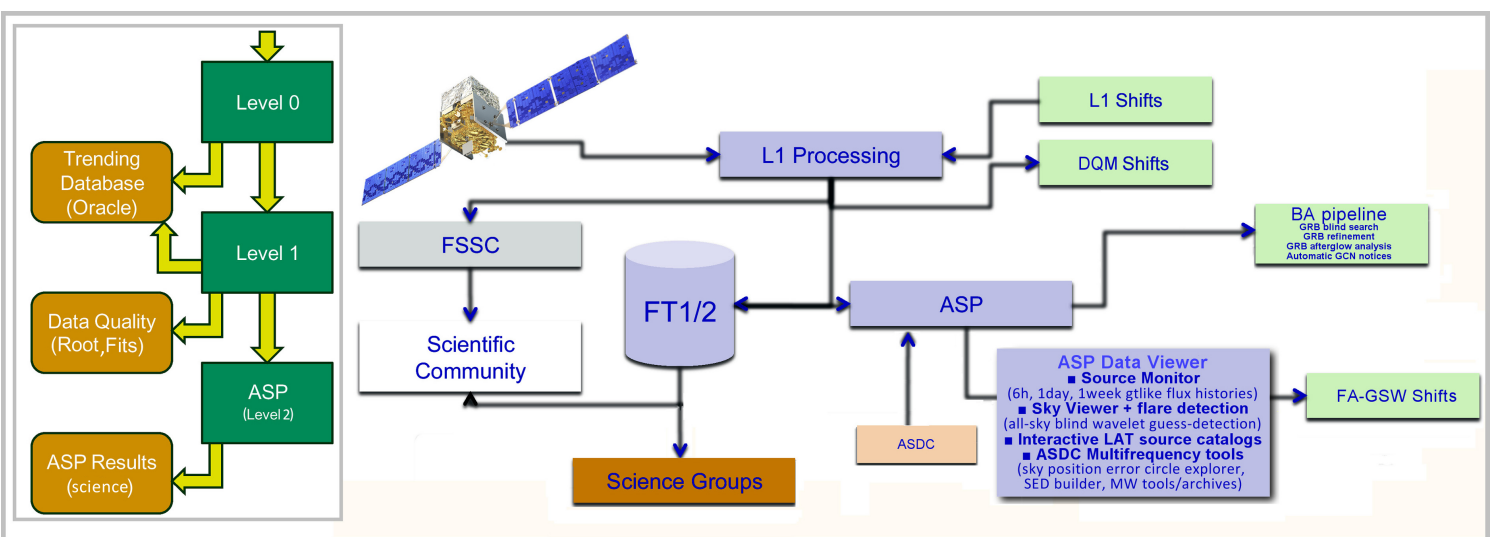

Figure 2: Left inset panel: diagram of the main general blocks of the Fermi LAT data processing flow at ISOC (SLAC, Stanford University). The astrophysical science data (FT1/FT2 fits files) are sent to the Fermi Science Support Center (FSSC) of the NASA Goddard Space Flight Center for public distribution to the scientific community. Main panel: block diagram of Fermi LAT data processing with final steps of duty services covered by members of the LAT collaboration in evidence (L1, Data Quality Monitor, DQM, Flare Advocate Gamma-ray Sky Watcher FA-GSW, weekly shifts). In particular the Automated Science Processing (ASP, Level 2 processing) is outlined with the links to the GRB automatic analysis pipeline and to the FA-GSW duty activity.

observed by the LAT, performed with continuity for all the year through weekly shifts.

The FA-GSW service points out basic facts and information about the $\gamma$-ray sky of potential interest for the LAT science groups, through a day-by-day inspection and review of the allsky photon count maps collected and of results of the quicklook science pipeline. Summaries about Fermi LAT detections of variable sources, transients, brightness trends, flaring sources, new sources on six-hour and 1-day intervals are communicated along with any relevant news to the external multiwavelength (MW) and TeV Cherenkov communities using the LAT-MW mailing-list ${ }^{1}$, posting Astronomer's Telegrams (ATels) ${ }^{2}$, automatic burst GCNs and special GCNs for blazar flares, and publishing weekly reports in the "Fermi Sky Blog"3. Thanks to this service joined with the public distribution of LAT data at the FSSC ${ }^{4}$ the Fermi LAT collaboration is therefore able to promote and increase the rate of multifrequency collaborations and observations, maximizing the scientific return and the rate of international scientific cooperation with this mission. First seeds for variability and MW follow-up observations and studies often are actually triggered by the FA-GSW service. More information can be found through the LAT MW Coordinating Group 5 .

\section{ASP infrastructure}

The FA-GSW duty is based on the automated quicklook data analysis of Level 2 (L2) at the Fermi LAT Instrument Science Operation Center (ISOC) of SLAC (Fig. 2). L2 processing (instrument monitoring pipeline, background monitoring, quick look science analysis) is triggered

\footnotetext{
${ }^{1}$ Sign up for "gammamw" mailing list at address: http://fermi.gsfc.nasa.gov/ssc/library/newsletter/

${ }^{2}$ Web address: http: //www. astronomerstelegram.org

${ }^{3}$ Web address: http://fermisky.blogspot.com

${ }^{4}$ Web address: http: //fermi.gsfc.nasa.gov/ssc/

${ }^{5}$ Web address: https://confluence.slac.stanford.edu/x/YQW
} 
Table 1: The 159 ATels posted by the Fermi LAT collaboration divided for topics.

\begin{tabular}{|l|c|}
\hline ATel type & Number \\
\hline Total Fermi ATels (on blazars mostly) & 159 \\
\hline Fermi on Galactic sources & 18 \\
Fermi on the Sun & 3 \\
Swift results on ToOs triggered by Fermi & 18 \\
Fermi-Swift joined results & 1 \\
Fermi-Integral joined results & 1 \\
Fermi-WEBT joined results & 1 \\
Fermi-HESS joined results & 1 \\
\hline
\end{tabular}
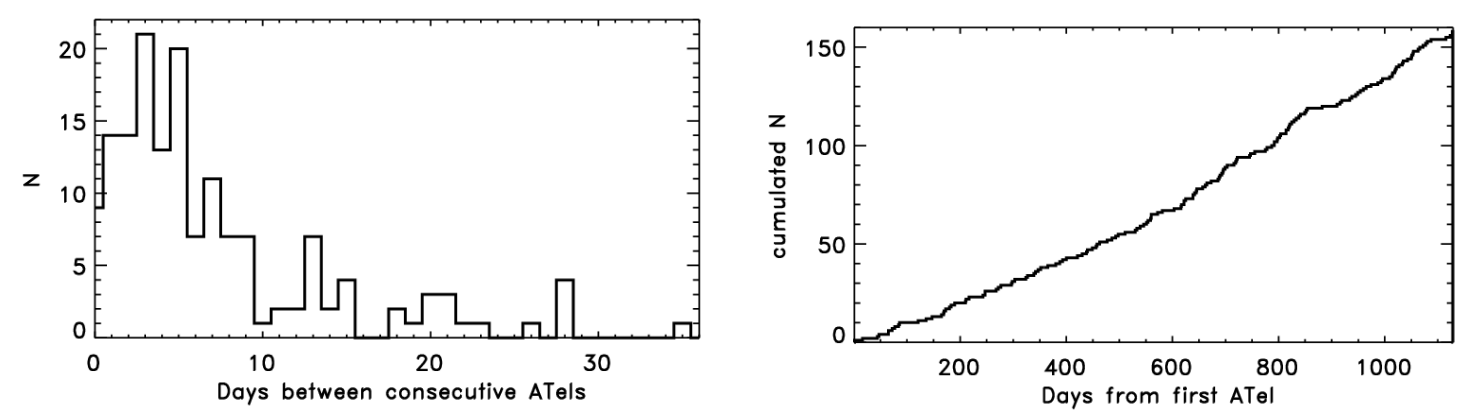

Figure 3: Distributions of the 159 Astronomical Telegrams (ATels) published on behalf of the Fermi LAT Collaboration from July 24, 2008 (ATel\#1628) to August 24, 2011 (ATel\#3580), i.e. in about 3 years.

by the first availability of Level 1 (L1) processed data and performed on longer time intervals (6 hours, 1 day , 1 week) referred therefore as Automated Science Processing (ASP). The ASP analysis pipeline running on the final astrophysical science data (photon event files FT1, and spacecraft data files FT2 fits files) is composed of several scientific tasks (Fig. 2, and Chiang (2007); Cameron (2007)): 1) automatic analysis of $\gamma$-ray bursts (impulsive transients) through refinement of parameters for LAT-detected GRBs, detection and characterization of GRBs not detected onboard, search and analysis of delayed high-energy afterglow emission; 2) flux history monitoring based on maximum-likelihood method ( $g$ t like science tool) of predefined list of sources (Data Release Plan, DRP, sources) with subsequent addictions of publicly announced sources (flaring blazars, sources in ATels); 3) blind guess-detection on all-sky photon counts maps accumulated in 6-hours, 1-day, 1-week intervals, through a fast method based on two-dimensional Mexican Hat wavelet transform, thresholding and sliding cell algorithms (Ciprini et al., 2007); 4) transient and flare identification based on variability test; 5) interactive LAT source catalogs; 6) multimission/multifrequency tools and archives (like the error circle explorer and spectral energy distribution builder) linked to ASP and provided by the ASI Science Data Center (ASDC ${ }^{6}$, Roma).

\section{Some results triggered by FA-GSW activity}

The role and activity of the FA-GSWs is twofold. 1) Gamma-ray Flare Advocate task: flaring sources approaching daily flux of $10^{-6}$ photons $\mathrm{cm}^{-2} \mathrm{~s}^{-1}$ deserves attention (detection, localization, flux, photon index checked, photon counts maps and exposure maps are outlooked). Internal/public notes, ATels, Target of Opportunity (ToO) are submitted, MW observing campaigns are

\footnotetext{
${ }^{6}$ Web address: http://fermi.asdc.asi.it
} 

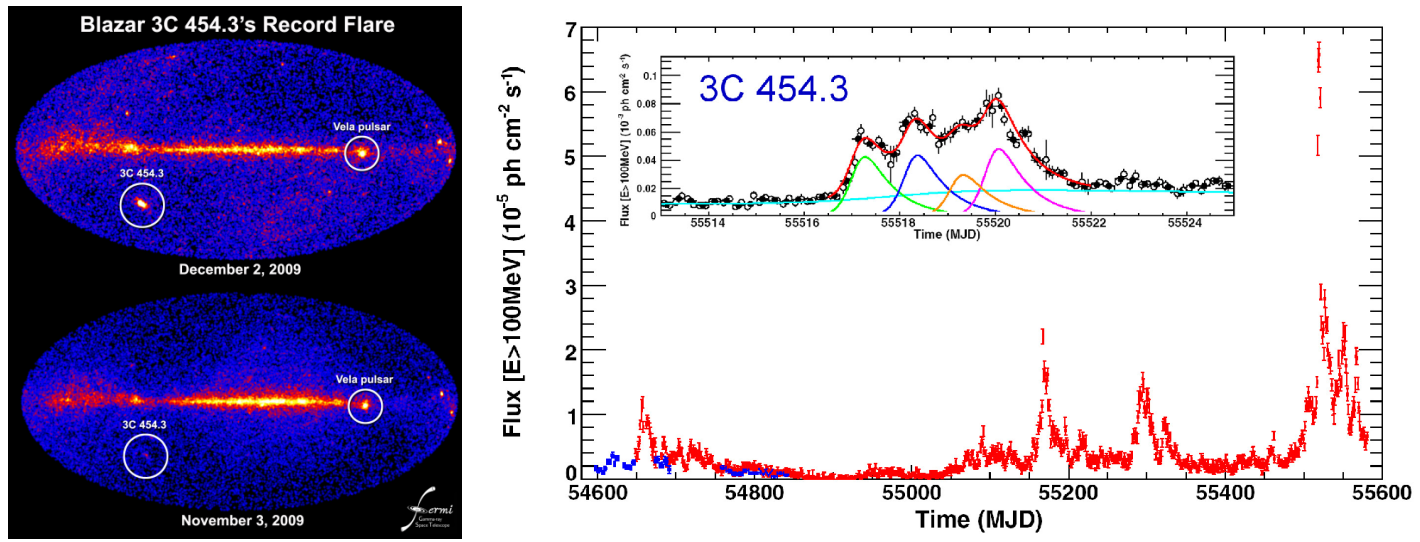

Figure 4: Left panel: examples of all-sky photon count map above $100 \mathrm{MeV}$ accumulated in 1 day of Fermi LAT survey. Variable all-sky maps like this and automatic quick-look source detections are investigated on 1-day and 6-hour time intervals day by day for all the year by Fermi Flare Advocates. The record's outburst of the $\gamma$-ray loud blazar 3C 454.3 is marked in comparison with the Vela pulsar source in two different days. Right panel: daily light curve of 3C454.3 measured with the Fermi-LAT since launch. Light curve of the flux above $100 \mathrm{MeV}$. Open and filled symbols are $3 \mathrm{hr}$ and $6 \mathrm{hr}$ averaged fluxes. Five component fit (sub-flares) is outlined (inset). Adapted from Abdo et al. (2011).

organized when needed. 2) Gamma-ray Sky Watcher task. Results from the ASP pipeline in 1-day and 6-hour time intervals are checked, searching for transients, increasing/decrasing brightness trends, and new $\gamma$-ray source candidates and looking at positional associations.

FA-GSWs discovered new $\gamma$-ray blazars before Fermi catalogs releases, discovered several bright flares from blazars, some transient from low galactic latitude sources, observed the emission of the quiet and flaring sun. FA-GSWs carried out also several targets of opportunity (ToO) to the Swift satellite (about a dozen per year), involved radio-astronomy community in joint observing programs, organized MW observing campaigns on several blazar and galactic source targets. In Table 1 and Fig. 3 basic statistics about the 159 Astronomical Telegrams (ATels) published on behalf of the Fermi LAT Collaboration from July 24, 2008 (ATel\#1628) to August 24, 2011 (ATel\#3580) are illustrated. The average rate of published ATels is about one per week/shift.

More in detail the substantial menu of discoveries triggered by the FA-GSW service is: many flares from $\gamma$-ray blazars (the extraordinary outbursts of 3C 454.3 Fig. 4 and Abdo et al. (2011) and 4C 21.35, large flares of PKS 1510-089, PKS 1830-211, AO 0235+164, PKS 1502+106, Fig. 5, 3C 279, 3C 273, PKS 1622-253, 3C 66A, etc.); short/long activity duty cycles of bright $\gamma$-ray blazars; unidentified transients near the Galactic plane (like J0910-5041, J0109+6134, Galactic center region) or associated to Galactic sources (like the Crab nebula, the nova V407 Cyg, Fig. 5 and Abdo et al. (2010b), the microquasar Cyg X-3, the binary star system 1FGL J1018.6-5856), intense $\mathrm{MeV}$ emission from the quiet and active sun.

Fermi LAT is demonstrating good capabilities in $\gamma$-ray variability monitoring and an optimal synergy with the Swift mission and radio-telescopes. The FA-GSW duty is complementing this all-sky surveying as the liaison between the LAT team and the MW astrophysical/astroparticle community, always invited to observe LAT sources and to propose new MW collaborations.

\section{Acknowledgments}

The Fermi LAT Collaboration acknowledges support from a number of agencies and institutes for both develop- 

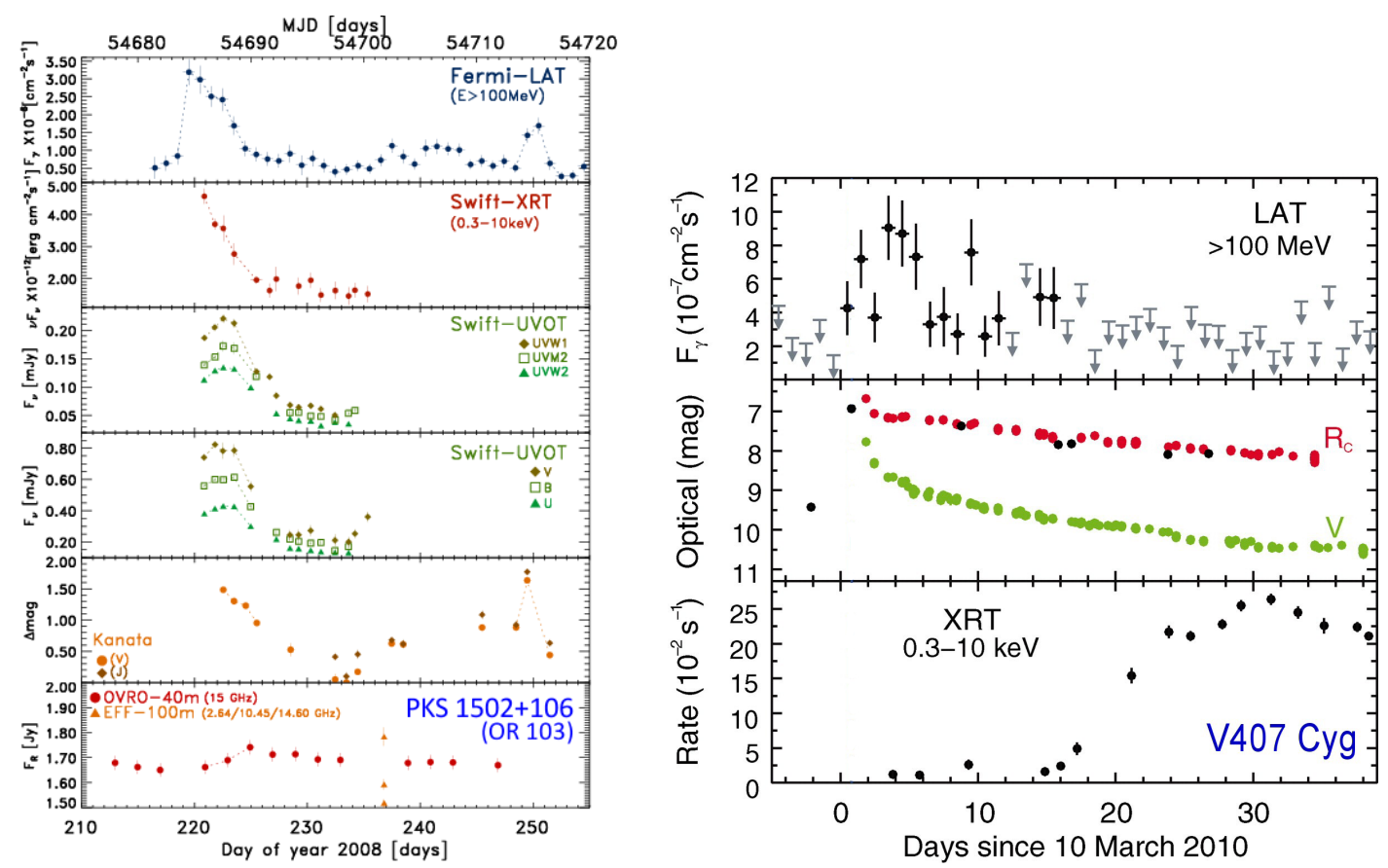

Figure 5: Left panel: Simultaneous $\gamma$-ray and MW light curves of the newly discovered $\gamma$-ray blazar PKS $1502+106$ (OR 103) obtained during the MW campaign triggered by the outburst detected by Fermi LAT in Aug. 2008. Data reported from above to the bottom are: Fermi-LAT (flux E $>100 \mathrm{MeV}$ ), Swift-XRT (0.3$10 \mathrm{keV}$ flux), Swift-UVOT (6-band optical-UV fluxes), Kanata-TRISPEC (optical-near-IR differential mag $\Delta V$ and $\Delta J$ bands), and OVRO $40 \mathrm{~m}$ (15 GHz flux). Adapted from Abdo et al. (2010c). Right panel: Light curves of V407 Cyg in $\gamma$-rays of Fermi -LAT (top), optical (middle), and Swift X-rays (bottom). Adapted from Abdo et al. (2010b).

ment and the operation of the LAT as well as scientific data analysis. These include NASA and DOE in the United States, CEA/Irfu and IN2P3/CNRS in France, ASI and INFN in Italy, MEXT, KEK, and JAXA in Japan, and the K. A. Wallenberg Foundation, the Swedish Research Council and the National Space Board in Sweden. Additional support from INAF in Italy and CNES in France for science analysis during the operations phase is also gratefully acknowledged.

\section{References}

Abdo, A. A., Ackermann, M., Ajello, M., et al. 2010a, ApJ, 722, 520

Abdo, A. A., Ackermann, M., Ajello, M., et al. 2010b, Science, 329, 817

Abdo, A. A., Ackermann, M., Ajello, M., et al. 2010c, ApJ, 710, 810

Abdo, A. A., Ackermann, M., Ajello, M., et al. 2011, ApJ Lett, 733, L26

Ackermann, M., Ajello, M., Allafort, A. et al., 2011, ApJ, 743, 171

Atwood, W. B., Abdo, A. A., Ackermann, M., et al. 2009, ApJ, 697, 1071

Cameron, R. A. 2007, AIP Conf Proc., 921, 534

Chiang, J. 2007, AIP Conf. Proc. 906, 11

Ciprini, S., Tosti, G., Marcucci, F., et al. 2007, AIP Conf. Proc., 921, 546 